\title{
Essential tremor with ubiquitinated Purkinje cell intranuclear inclusions
}

\author{
Elan D. Louis · Cordelia Erickson-Davis · Rajesh Pahwa • \\ Kelly E. Lyons · Anthony Garber · Carol B. Moskowitz • \\ Arlene Lawton · Phyllis L. Faust · Jean-Paul G. Vonsattel
}

Received: 3 December 2009/Revised: 11 January 2010/Accepted: 13 January 2010/Published online: 28 January 2010

(c) Springer-Verlag 2010

Despite intensive efforts to collect essential tremor (ET) brains, the number of postmortem examinations remains limited, and the range of pathological changes associated with ET has not been fully catalogued [3]. We report an ET patient who had a pattern of pathological change not previously reported in ET or another neurological disease.

The patient had childhood-onset, familial, kinetic arm tremor that progressively worsened during adulthood. She experienced difficulty eating and writing and developed head tremor. The clinical diagnosis was ET (see Supplementary online materials). She died at age 79 of myocardial infarction.

The brain was removed and placed on ice $6 \mathrm{~h}$ after death. At the New York Brain Bank, Columbia University,

Electronic supplementary material The online version of this article (doi:10.1007/s00401-010-0641-x) contains supplementary material, which is available to authorized users.

E. D. Louis - C. Erickson-Davis - A. Lawton

GH Sergievsky Center, College of Physicians and Surgeons, Columbia University, New York, NY, USA

E. D. Louis - C. B. Moskowitz

Department of Neurology, College of Physicians and Surgeons, Columbia University, New York, NY, USA

E. D. Louis · A. Lawton - J.-P. G. Vonsattel

Taub Institute for Research on Alzheimer's Disease

and the Aging Brain, College of Physicians and Surgeons,

Columbia University, New York, NY, USA

E. D. Louis

Department of Epidemiology, Mailman School of Public Health, Columbia University, New York, NY, USA

R. Pahwa $\cdot$ K. E. Lyons

Department of Neurology, University of Kansas Medical Center,

Kansas City, KS, USA external examination (J.P.G.V.) revealed mild (1+) frontal and parietal atrophy and two small, bilateral striatal infarcts. As described [3], blocks were taken from standardized brain regions and embedded in paraffin; 7- $\mu \mathrm{m}$ thick sections were stained with Luxol fast blue counterstained with hematoxylin-eosin (LH\&E). Additional sections from selected blocks were stained with modified Bielschowsky silver stain or antibodies directed against alpha-synuclein, beta-amyloid, hyperphosphorylated tau (AT8), glial fibrillary acidic protein (GFAP), ubiquitin, $1 \mathrm{C} 2$, and TDP-43. Torpedoes in one entire $20 \times 25 \mathrm{~mm}$ LH\&E-stained parasagittal cerebellar cortical section and another entire Bielschowsky-stained section were counted [3]. Bergmann cells were assessed (GFAP-immunostained section).

In the cerebellum, there were abundant torpedoes [18 (LH\&E) and 29 (Bielschowsky)] (Fig. 1a) and segmental

\author{
A. Garber \\ Comprehensive Genetics, Molecular Diagnostic Laboratory, \\ Milwaukee, WI, USA \\ P. L. Faust - J.-P. G. Vonsattel \\ Department of Pathology and Cell Biology, \\ College of Physicians and Surgeons, \\ Columbia University, New York, NY, USA \\ E. D. Louis $(\bowtie)$ \\ Unit 198, Neurological Institute, \\ 710 West 168th Street, New York, NY 10032, USA \\ e-mail: EDL2@columbia.edu
}




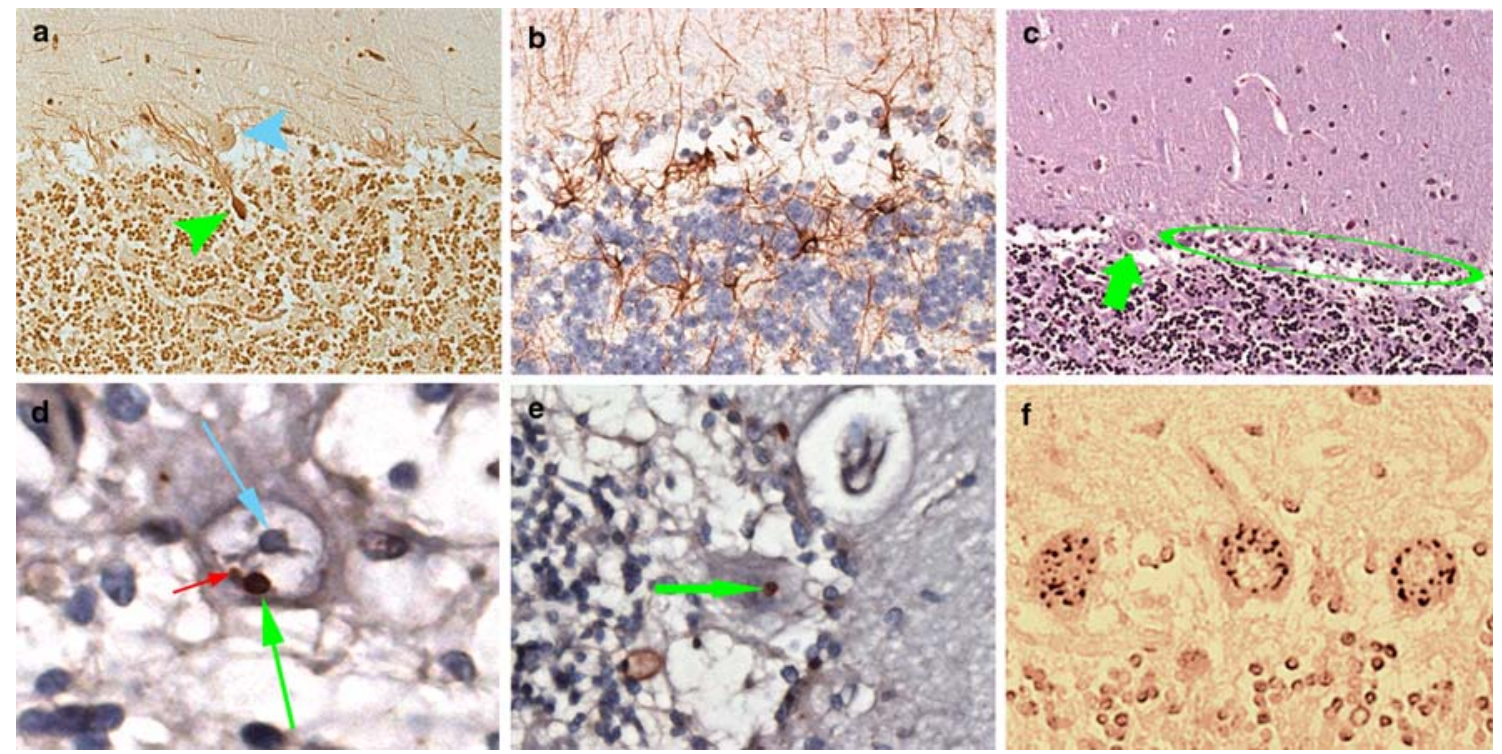

Fig. 1 a Torpedo (green arrow) associated with a PC (blue arrow) (Bielschowsky, 200× magnification). b Bergmann gliosis (GFAP, $400 \times$ magnification). c PC (green arrow) with adjacent segmental loss of PCs and Bergmann gliosis (green circle) (LH\&E, 200× magnification). d PC ubiquitinated intranuclear inclusion (green arrow) adjacent to nucleolus (blue arrow). A smaller ubiquitinated inclusion is also visualized (red arrow) (ubiquitin, 950× magnification). e PC ubiquitinated intranuclear inclusion (green arrow) (ubiquitin, $630 \times$ magnification). f $1 \mathrm{C} 2$ immunoreactivity is visualized in PC cytoplasm $(630 \times$ magnification) loss of Purkinje cells (PCs) with Bergmann gliosis (Fig. 1b, c). The dentate nucleus was normal. Throughout the section, PCs contained one or two prominent ubiquitinated, nuclear inclusions that could clearly be distinguished from the nucleoli (Fig. 1d, e). These inclusions, observed in approximately $3 \%$ of PCs, were not observed in astrocytes, oligodendrocytes, or neurons aside from PCs. Ubiquitinated inclusions were not observed on sections of the medulla, pons, superior frontal cortex, hippocampal formation, entorhinal region, caudate, putamen, nucleus accumbens, or globus pallidum. In the substantia nigra, rare neurons contained Marinesco bodies. 1C2 immunoreactivity in the PC cytoplasm indicated the formation of polyglutamine aggregates (Fig. 1f). TDP-43 immunoreactivity was normal.

On LH\&E- and alpha-synuclein-immunostained sections, no Lewy bodies or Lewy neurites were evident in the dorsal vagal nucleus, inferior olivary nucleus, locus ceruleus or substantia nigra.

Argyrophilic neuronal tangles or AT8-labeled neurons and threads were present throughout the cerebral cortex, with the involvement greatest in the entorhinal cortex, parahippocampal and occipitotemporal gyri, and temporal pole. Up to 20 neuritic plaques $/ 100 \times$ microscopic field were found in the motor cortex. In the hippocampal formation, up to 5 neuritic plaques $/ 100 \times$ microscopic field were found in the Sommer sector and subiculum. The Braak and Braak [1] Alzheimer's disease (AD) stage was VI.
Postmortem testing of frozen brain tissue was negative for fragile $\mathrm{X}$ tremor ataxia syndrome (FXTAS) and spinocerebellar ataxia (SCA) 1, 2, 3 and 6.

In recent postmortem studies, two subtypes of ET seem to have emerged [3]. The majority of ET brains have degenerative changes, including PC loss [3], in the cerebellum (i.e., "cerebellar ET"). A smaller number of brains have Lewy bodies in the brainstem with relatively normal cerebella (i.e., "Lewy body variant of ET") [3]. We now report what seems to be a third pattern of change, namely, PCs with ubiquitinated nuclear inclusions with cerebellar pathology.

The pattern of changes observed in this ET brain is likely to be rare, as it occurred in only 1 in 46 ET brains prospectively collected at our brain bank to date. Nevertheless, the current case is important for several reasons. First, it again demonstrates the pathological heterogeneity of ET and, by implication, suggests that ET is either a family of diseases or a syndrome. Second, it is of particular interest that the nuclear inclusions in this case were confined to the cerebellum. As in cerebellar ET, the main form of ET, the localization of pathological changes is in the cerebellum and postmortem changes are not observed in other brain regions. This serves to further confirm the importance of the cerebellum as the anatomical localization of the pathological changes in most cases of ET. Finally, as with the other two subtypes of ET (cerebellar ET and Lewy body variant of ET), the changes are of a 
degenerative nature, thereby, further reinforcing the notion of this disease as neurodegenerative.

The observation of ubiquitinated protein inclusions is one of the hallmarks of neurodegeneration. Ubiquitinated intranuclear inclusions are found in a variety of neurodegenerative diseases including FXTAS, SCAs, and Huntington's disease; however, in these disorders inclusions are rare or not found in the PCs, but present in widespread brain regions, and accompanied by marked additional changes on postmortem [2].

Our patient did not have FXTAS as the inclusions were limited to the PCs and genetic testing was negative. Furthermore, an ET-like phenotype is very rare in that disorder. Against a diagnosis of SCA is the clinical presentation (kinetic tremor rather than intention tremor, absence of other cerebellar signs), negative genetic test results, and postmortem findings (e.g., absence of: pontine atrophy or neuronal loss in inferior olivary nucleus or more complete loss of PCs).

Our patient developed $\mathrm{AD}$ in the final year of life. $\mathrm{AD}$ patients do not have ubiquitinated intranuclear inclusions in the PCs or elsewhere, the exception being the Marinesco bodies within the scattered neurons of the substantia nigra pars compacta.
In summary, this ET patient had a pattern of pathological change that has not been reported previously. This case further reinforces the notion that ET is likely to be a family of degenerative diseases whose pathology is usually centered in the cerebellum.

Acknowledgments R01 NS42859 from the National Institutes of Health (Bethesda, MD); the Parkinson's Disease Foundation (New York, NY).

Conflict of interest statement The authors report no conflicts of interest.

\section{References}

1. Braak H, Alafuzoff I, Arzberger T, Kretzschmar H, Del Tredici K (2006) Staging of Alzheimer disease-associated neurofibrillary pathology using paraffin sections and immunocytochemistry. Acta Neuropathol 112:389-404

2. Louis E, Moskowitz C, Friez M, Amaya M, Vonsattel JP (2006) Parkinsonism, dysautonomia, and intranuclear inclusions in a fragile X carrier: a clinical-pathological study. Mov Disord 21:420-425

3. Louis ED, Faust PL, Vonsattel JP et al (2007) Neuropathological changes in essential tremor: 33 cases compared with 21 controls. Brain 130:3297-3307 